\title{
DIFFERENTIAL INVARIANTS OF TWO AFFINE CURVE FAMILIES
}

\author{
YASEmin SAĞIroĞLU ${ }^{1}$, Demet Aydemir ${ }^{1}$, UĞUR GÖzÜtoK ${ }^{1, *}$ \\ ${ }^{1}$ Karadeniz Technical University \\ Department of Mathematics \\ 61080 Trabzon, Turkey
}

E-mails: sagiroglu.yasemin@gmail.com, aydemirdemet61@gmail.com, ugurgozutok@ktu.edu.tr

\begin{abstract}
This paper examines the affine differential invariants of two curves. The equivalence of two curves is obtained by using these invariants according to the affine group. In addition, obtained differential invariants will be shown to be the minimal system of the generators.

Key words and Phrases: Affine group; differential invariants; equivalence.
\end{abstract}

\begin{abstract}
Abstrak. Paper ini membahas tentang invariant affine diferensial dari dua kurva. Dengan menggunakan invarian ini, diperoleh ekivalensi antara dua kurva berdasarkan pada grup affine. Lebih jauh, ditunjukkan bahwa invarian diferensial yang diperoleh merupakan sistem pembangun minimal.

Key words and Phrases: Grup affine; invarian diferensial; ekivalensi.
\end{abstract}

\section{INTRODUCTION}

The notion of affine differential geometry arose from Felix Klein's Erlangen Program in 1872. According to this program, affine differential geometry consists of properties which are invariant under the affine transformations. In affine differential geometry, studies have been done about affine invariants and generators of affine invariants. The construction of affine invariants of curves was studied in [8-11]. Based on this, solution of the equivalence problem has been studied also.

Differential geometry of curves has been studied for many years. It's been studied in many aspects in the subgroups of the affine group. In some of these

2000 Mathematics Subject Classification: 53A15, 53A55.

Received: 28-04-2017, revised: 13-07-2018, accepted: 13-07-2018.

* Corresponding Author 
studies, invariants such as arc-length, curvature have been examined. In [1], centroaffine invariants, arc length and curvature functions of a curve in affine $n$-space are obtained. In addition, several authors studied the affine curves and their invariants in several works $[2,3,6]$.

Equivalence of curves in $S L(n, \mathbb{R})$ is given in [5]. Equivalence problem for parametric curves in one dimensional affine space is studied in [4]. In this study, affine differential invariant system for two curves is studied and by using this system, equivalence of a curve family which consists of two curves is given. Also, it is shown that the affine differential invariant system of this curve family is minimal.

\section{Preliminaries}

For two affine curves $x_{1}, x_{2}$ a differential polynomial of these curves is given by $P\left\{x_{1}, x_{2}\right\}=P\left(x_{1}, x_{2}, x_{1}^{\prime}, x_{2}^{\prime}, \ldots, x_{1}^{(m)}, x_{2}^{(m)}\right)$ for some natural number $m$, where for $k \in \mathbb{N}, x_{i}^{(k)}$ is the $k^{t h}$ derivative of $x_{i}$. Function $f<x_{1}, x_{2}>=\frac{P_{1}\left\{x_{1}, x_{2}\right\}}{P_{2}\left\{x_{1}, x_{2}\right\}}$ such that $P_{2}\left\{x_{1}, x_{2}\right\} \neq 0$ is called a differential rational function. The set of differential rational functions is denoted by $\mathbb{R}<x_{1}, x_{2}>$.

For an element $F \in \operatorname{Aff}(2, \mathbb{R})$, if $f<F x_{1}, F x_{2}>=f<x_{1}, x_{2}>$ then the function $f$ is called a $A f f(2, \mathbb{R})$-invariant differential rational function. The set of all $\operatorname{Aff}(2, \mathbb{R})$-invariant differential rational functions is denoted by $\mathbb{R}<$ $x_{1}, x_{2}>^{A f f(2, \mathbb{R})} . \mathbb{R}<x_{1}, x_{2}>^{A f f(2, \mathbb{R})}$ is a differential subfield and a sub $\mathbb{R}$-algebra of $\mathbb{R}<x_{1}, x_{2}>$.

Lemma 2.1. For vectors $x_{0}, x_{1}, \ldots, x_{n}, y_{2}, \ldots, y_{n}$ in $\mathbb{R}^{n}$ following equation holds: $\left[x_{1} x_{2} \ldots x_{n}\right]\left[x_{0} y_{2} \ldots y_{n}\right]-\left[x_{0} x_{2} \ldots x_{n}\right]\left[x_{1} y_{2} \ldots y_{n}\right]-\ldots-\left[x_{1} x_{2} \ldots x_{0}\right]\left[x_{n} y_{2} \ldots y_{n}\right]=0$

Proof. A sketch of the proof is in [2].

\section{Main Theorems And Definitions}

Definition 3.1. For a curve $x_{1}$ in $\mathbb{R}^{2}$, if determinant $\left[x_{1}^{\prime} x_{1}^{\prime \prime}\right] \neq 0$ then $x_{1}$ is called Aff $(2, \mathbb{R})$-regular curve.

Theorem 3.2. For curves $x_{1}, x_{2}$ in $\mathbb{R}^{2}$ where $x_{1}$ is Aff $(2, \mathbb{R})$-regular, generator system of $\mathbb{R}<x_{1}, x_{2}>^{A f f(2, \mathbb{R})}$ is as follows:

$$
\frac{\left[x_{1}^{\prime} x_{1}^{\prime \prime \prime}\right]}{\left[x_{1}^{\prime} x_{1}^{\prime \prime}\right]}, \frac{\left[x_{1}^{\prime \prime \prime} x_{1}^{\prime \prime}\right]}{\left[x_{1}^{\prime} x_{1}^{\prime \prime}\right]}, \frac{\left[x_{2}-x_{1} x_{1}^{\prime \prime}\right]}{\left[x_{1}^{\prime} x_{1}^{\prime \prime}\right]}, \frac{\left[x_{1}^{\prime} x_{2}-x_{1}\right]}{\left[x_{1}^{\prime} x_{1}^{\prime \prime}\right]} \text {. }
$$

Proof. Let $f \in \mathbb{R}<x_{1}, x_{2}>^{A f f(2, \mathbb{R})}$. Then there exist an element $k \in \mathbb{N}$ such that

$$
f<x_{1}, x_{2}>=f<x_{1}, x_{2}, x_{1}^{\prime}, x_{2}^{\prime}, \ldots, x_{1}^{(k)}, x_{2}^{(k)}>.
$$

For any $g \in \operatorname{Aff}(2, \mathbb{R})$ and $b \in \mathbb{R}^{2}, f<g x_{1}+b, g x_{2}+b>=f<x_{1}, x_{2}>$. Hence we have

$$
\begin{gathered}
f<g x_{1}+b, g x_{2}+b, g x_{1}^{\prime}+b, g x_{2}^{\prime}+b, \ldots, g x_{1}^{(k)}+b, g x_{2}^{(k)}+b>=f< \\
x_{1}, x_{2}, x_{1}^{\prime}, x_{2}^{\prime}, \ldots, x_{1}^{(k)}, x_{2}^{(k)}>.
\end{gathered}
$$


If we put $g=e$, then

$$
\begin{gathered}
f<x_{1}+b, x_{2}+b, x_{1}^{\prime}+b, x_{2}^{\prime}+b, \ldots, x_{1}^{(k)}+b, x_{2}^{(k)}+b>=f< \\
x_{1}, x_{2}, x_{1}^{\prime}, x_{2}^{\prime}, \ldots, x_{1}^{(k)}, x_{2}^{(k)}>.
\end{gathered}
$$

Since above equation holds for any $b$, it holds for $b=-x_{1}$. Therefore

$$
f<x_{1}+b, x_{2}+b, x_{1}^{\prime}, x_{2}^{\prime}, \ldots, x_{1}^{(k)}, x_{2}^{(k)}>=\varphi<x_{2}-x_{1}, x_{1}^{\prime}, x_{2}^{\prime}, \ldots, x_{1}^{(k)}, x_{2}^{(k)}>.
$$

Since $\varphi$ is $G L(2, \mathbb{R})$-invariant, following equation holds:

$$
\varphi<g\left(x_{2}-x_{1}\right), g x_{1}^{\prime}, g x_{2}^{\prime}, \ldots, g x_{1}^{(k)}, g x_{2}^{(k)}>=\varphi<x_{2}-x_{1}, x_{1}^{\prime}, x_{2}^{\prime}, \ldots, x_{1}^{(k)}, x_{2}^{(k)}>.
$$

If $x_{2}-x_{1}=y_{2}$ and $x_{1}^{\prime}=y_{1}$, then for any $g \in G L(2, \mathbb{R})$ following equation holds:

$$
\psi<g y_{1}, g y_{2}, g y_{1}^{\prime}, g y_{2}^{\prime}, \ldots, g y_{2}^{(k)}>=\psi<y_{1}, y_{2}, y_{1}^{\prime}, y_{2}^{\prime}, \ldots, y_{2}^{(k)}>.
$$

Since $\psi$ is $G L(2, \mathbb{R})$-invariant, following generators are obtained [7] :

$$
\frac{\left[\begin{array}{ll}
y_{1}^{\prime \prime} & y_{1}^{\prime}
\end{array}\right]}{\left[\begin{array}{ll}
y_{1} & y_{1}^{\prime}
\end{array}\right]}, \frac{\left[\begin{array}{ll}
y_{1} & y_{1}^{\prime \prime}
\end{array}\right]}{\left[\begin{array}{ll}
y_{1} & y_{1}^{\prime}
\end{array}\right]}, \frac{\left[\begin{array}{ll}
y_{2} & y_{1}^{\prime}
\end{array}\right]}{\left[\begin{array}{ll}
y_{1} & y_{1}^{\prime}
\end{array}\right]}, \frac{\left[\begin{array}{ll}
y_{1} & y_{2}
\end{array}\right]}{\left[\begin{array}{ll}
y_{1} & y_{1}^{\prime}
\end{array}\right]} .
$$

If we substitute $x_{2}-x_{1}=y_{2}$ and $x_{1}^{\prime}=y_{1}$ in the above terms, we have

$$
\frac{\left[x_{1}^{\prime} x_{1}^{\prime \prime \prime}\right]}{\left[x_{1}^{\prime} x_{1}^{\prime \prime}\right]}, \frac{\left[x_{1}^{\prime \prime \prime} x_{1}^{\prime \prime}\right]}{\left[x_{1}^{\prime} x_{1}^{\prime \prime}\right]}, \frac{\left[x_{2}-x_{1} x_{1}^{\prime \prime}\right]}{\left[x_{1}^{\prime} x_{1}^{\prime \prime}\right]}, \frac{\left[x_{1}^{\prime} x_{2}-x_{1}\right]}{\left[x_{1}^{\prime} x_{1}^{\prime \prime}\right]} \text {. }
$$

This completes the proof.

Definition 3.3. Let $\left\{x_{1}, x_{2}\right\}$ and $\left\{y_{1}, y_{2}\right\}$ be two curve families such that $x_{i}, y_{i}$ : $I \subset \mathbb{R} \rightarrow \mathbb{R}^{2}, i=1,2$ and $G=\operatorname{Aff}(2, \mathbb{R})$. If there exist an element $g \in G L(2, \mathbb{R})$ and $b \in \mathbb{R}^{2}$ such that $g x_{i}(t)+b=y_{i}(t)$ for all $t \in I$ and $i=1,2$ then the curve families $\left\{x_{1}, x_{2}\right\}$ and $\left\{y_{1}, y_{2}\right\}$ are said to be Aff $(2, \mathbb{R})$-equivalent. Aff $(2, \mathbb{R})$ equivalence is denoted by $\left\{x_{1}, x_{2}\right\} \stackrel{\mathrm{G}}{\approx}\left\{y_{1}, y_{2}\right\}$.

Theorem 3.4. Let $G=\operatorname{Aff}(2, \mathbb{R})$ and $\left\{x_{1}, x_{2}\right\}$ and $\left\{y_{1}, y_{2}\right\}$ be two curve families where $x_{1}$ and $y_{1}$ are Aff $(2, \mathbb{R})$-regular. If

$$
\begin{gathered}
\frac{\left[x_{1}^{\prime} x_{1}^{\prime \prime \prime}\right]}{\left[x_{1}^{\prime} x_{1}^{\prime \prime}\right]}=\frac{\left[y_{1}^{\prime} y_{1}^{\prime \prime \prime}\right]}{\left[y_{1}^{\prime} y_{1}^{\prime \prime}\right]}, \frac{\left[x_{1}^{\prime \prime \prime} x_{1}^{\prime \prime}\right]}{\left[x_{1}^{\prime} x_{1}^{\prime \prime}\right]}=\frac{\left[y_{1}^{\prime \prime \prime} y_{1}^{\prime \prime}\right]}{\left[y_{1}^{\prime} y_{1}^{\prime \prime}\right]} \\
\frac{\left[x_{2}-x_{1} x_{1}^{\prime \prime}\right]}{\left[x_{1}^{\prime} x_{1}^{\prime \prime}\right]}=\frac{\left[y_{2}-y_{1} y_{1}^{\prime \prime}\right]}{\left[y_{1}^{\prime} y_{1}^{\prime \prime}\right]}, \frac{\left[x_{1}^{\prime} x_{2}-x_{1}\right]}{\left[x_{1}^{\prime} x_{1}^{\prime \prime}\right]}=\frac{\left[y_{1}^{\prime} y_{2}-y_{1}\right]}{\left[y_{1}^{\prime} y_{1}^{\prime \prime}\right]}
\end{gathered}
$$

then $\left\{x_{1}, x_{2}\right\} \stackrel{G}{\approx}\left\{y_{1}, y_{2}\right\}$.

Proof. Assume that $x_{1}^{\prime}=z_{1}, y_{1}^{\prime}=w_{1}, x_{2}-x_{1}=z_{2}$ and $y_{2}-y_{1}=w_{2}$. Then we have

$$
\frac{\left[z_{1} z_{1}^{\prime \prime}\right]}{\left[z_{1}^{\prime} z_{1}^{\prime}\right]}=\frac{\left[w_{1} w_{1}^{\prime \prime}\right]}{\left[w_{1} w_{1}^{\prime}\right]}, \frac{\left[z_{1}^{\prime} z_{1}^{\prime \prime}\right]}{\left[z_{1} z_{1}^{\prime}\right]}=\frac{\left[w_{1}^{\prime} w_{1}^{\prime \prime}\right]}{\left[w_{1} w_{1}^{\prime}\right]}
$$




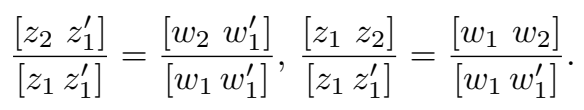

Consider the following matrices:

$$
A_{z}=\left(\begin{array}{cc}
z_{11}(t) & z_{11}^{\prime}(t) \\
z_{12}(t) & z_{12}^{\prime}(t)
\end{array}\right), \quad A_{z}^{\prime}=\left(\begin{array}{cc}
z_{11}^{\prime}(t) & z_{11}^{\prime \prime}(t) \\
z_{12}^{\prime}(t) & z_{12}^{\prime \prime}(t)
\end{array}\right)
$$

Since $\left[\begin{array}{ll}z_{1} & z_{1}^{\prime}\end{array}\right]=\left[\begin{array}{ll}x_{1}^{\prime} & x_{1}^{\prime \prime}\end{array}\right] \neq 0$, the matrix $A_{z}$ is invertible. If $A_{z}^{-1} A_{z}^{\prime}=C$, then $A_{z}^{\prime}=A_{z} C$. Therefore

$$
\left(\begin{array}{ll}
z_{11}^{\prime} & z_{11}^{\prime \prime} \\
z_{12}^{\prime} & z_{12}^{\prime \prime}
\end{array}\right)=\left(\begin{array}{ll}
z_{11} & z_{11}^{\prime} \\
z_{12} & z_{12}^{\prime}
\end{array}\right)\left(\begin{array}{ll}
c_{11} & c_{12} \\
c_{21} & c_{22}
\end{array}\right)
$$

From the above equation, we have the following equation system:

$$
\begin{aligned}
& z_{11}^{\prime}=c_{11} z_{11}+c_{21} z_{11}^{\prime} \\
& z_{12}^{\prime}=c_{11} z_{12}+c_{21} z_{12}^{\prime} \\
& z_{11}^{\prime \prime}=c_{12} z_{11}+c_{22} z_{11}^{\prime} \\
& z_{12}^{\prime \prime}=c_{12} z_{12}+c_{22} z_{12}^{\prime} .
\end{aligned}
$$

By solving this equation system, we have

$$
c_{11}=0, \quad c_{21}=1, \quad c_{12}=\frac{\left[z_{1}^{\prime} z_{1}^{\prime \prime}\right]}{\left[z_{1} z_{1}^{\prime}\right]}, \quad c_{22}=\frac{\left[z_{1} z_{1}^{\prime \prime}\right]}{\left[z_{1} z_{1}^{\prime}\right]} .
$$

Similarly, for the matrices

$$
A_{w}=\left(\begin{array}{ll}
w_{11}(t) & w_{11}^{\prime}(t) \\
w_{12}(t) & w_{12}^{\prime}(t)
\end{array}\right), \quad A_{w}^{\prime}=\left(\begin{array}{cc}
w_{11}^{\prime}(t) & w_{11}^{\prime \prime}(t) \\
w_{12}^{\prime}(t) & w_{12}^{\prime \prime}(t)
\end{array}\right),
$$

assume that $A_{w}^{-1} A_{w}^{\prime}=D$. Hence,

$$
\begin{aligned}
\left(A_{w} A_{z}^{-1}\right)^{\prime} & =A_{w}^{\prime} A_{z}^{-1}+A_{w}\left(A_{z}^{-1}\right)^{\prime} \\
& =A_{w}^{\prime} A_{z}^{-1}-A_{w} A_{z}^{-1} A_{z}^{\prime} A_{z}^{-1} \\
& =A_{w} A_{w}^{-1} A_{w}^{\prime} A_{z}^{-1}-A_{w} A_{z}^{-1} A_{z}^{\prime} A_{z}^{-1} \\
& =A_{w}\left(A_{w}^{-1} A_{w}^{\prime}-A_{z}^{-1} A_{z}^{\prime}\right) A_{z}^{-1}=0 .
\end{aligned}
$$

From the above equation, there exist an element $g \in G L(2, \mathbb{R})$ such that $A_{w} A_{z}^{-1}=$ $g$. Therefore we have $A_{w}=g A_{z}$ that means

$$
\left(\begin{array}{ll}
w_{11} & w_{11}^{\prime} \\
w_{12} & w_{12}^{\prime}
\end{array}\right)=\left(\begin{array}{ll}
g_{11} & g_{12} \\
g_{21} & g_{22}
\end{array}\right)\left(\begin{array}{ll}
z_{11} & z_{11}^{\prime} \\
z_{12} & z_{12}^{\prime}
\end{array}\right)
$$

In that case, for any $t$ we have $w_{1}(t)=g z_{1}(t)$ which means $y_{1}^{\prime}(t)=x_{1}^{\prime}(t)$. By integrating both side of this equation, following equation is obtained:

$$
y_{1}(t)=g x_{1}(t)+b .
$$

In the same way consider the following matrices: 


$$
D_{z}=\left(\begin{array}{cc}
z_{11}(t) & z_{21}(t) \\
z_{12}(t) & z_{22}(t)
\end{array}\right), \quad D_{z}^{\prime}=\left(\begin{array}{cc}
z_{11}^{\prime}(t) & z_{21}^{\prime}(t) \\
z_{12}^{\prime}(t) & z_{22}^{\prime}(t)
\end{array}\right) .
$$

Assume that $A_{z}^{-1} D_{z}=H$, then $D_{z}=A_{z} H$. From this equation, we have:

$$
\left(\begin{array}{ll}
z_{11} & z_{21} \\
z_{12} & z_{22}
\end{array}\right)=\left(\begin{array}{ll}
z_{11} & z_{11}^{\prime} \\
z_{12} & z_{12}^{\prime}
\end{array}\right)\left(\begin{array}{ll}
h_{11} & h_{12} \\
h_{21} & h_{22}
\end{array}\right)
$$

Hence the following equation system holds:

$$
\begin{aligned}
& z_{11}=h_{11} z_{11}+h_{21} z_{11}^{\prime} \\
& z_{12}=h_{11} z_{12}+h_{21} z_{12}^{\prime} \\
& z_{21}=h_{12} z_{11}+h_{22} z_{11}^{\prime} \\
& z_{22}=h_{12} z_{12}+h_{22} z_{12}^{\prime} .
\end{aligned}
$$

By solving this equation system, following solutions are obtained:

$$
h_{11}=1, \quad h_{21}=0, \quad h_{12}=\frac{\left[z_{2} z_{1}^{\prime}\right]}{\left[z_{1} z_{1}^{\prime}\right]}, \quad h_{22}=\frac{\left[z_{1} z_{2}\right]}{\left[z_{1} z_{1}^{\prime}\right]} .
$$

If we use $A_{w}=g A_{z}$ in the equation $A_{z}^{-1} D_{z}=H=A_{w}^{-1} D_{w}$, we have $A_{z}^{-1} D_{z}=$ $\left(g A_{z}\right)^{-1} D_{w}=A_{z}^{-1} g^{-1} D_{w}$ that means $D_{z}=g^{-1} D_{w}$ and $D_{w}=g D_{z}$. Matrix form of the last equation is

$$
\left(\begin{array}{ll}
w_{11} & w_{21} \\
w_{12} & w_{22}
\end{array}\right)=\left(\begin{array}{ll}
g_{11} & g_{12} \\
g_{21} & g_{22}
\end{array}\right)\left(\begin{array}{ll}
z_{11} & z_{21} \\
z_{12} & z_{22}
\end{array}\right)
$$

From this matrix equation, $w_{2}=g z_{2}$ that means $y_{2}-y_{1}=g\left(x_{2}-x_{1}\right)$. In that case we have

$$
y_{2}=g x_{2}+b
$$

Equations (1) and (2) complete the proof.

Theorem 3.5. Let $G=\operatorname{Aff}(2, \mathbb{R})$ and $f_{1}(t), f_{2}(t), f_{3}(t), f_{4}(t)$ be $C^{\infty}$-functions such that $t \in I \subset \mathbb{R}$. There exists a curve family $\left\{x_{1}, x_{2}\right\}$ such that $x_{1}$ is $G L(n, \mathbb{R})-$ regular, which satisfies the following equations:

$$
\begin{gathered}
\frac{\left[x_{1}^{\prime} x_{1}^{\prime \prime \prime}\right]}{\left[x_{1}^{\prime} x_{1}^{\prime \prime}\right]}=f_{1}(t), \frac{\left[x_{1}^{\prime \prime \prime} x_{1}^{\prime \prime}\right]}{\left[x_{1}^{\prime} x_{1}^{\prime \prime}\right]}=f_{2}(t), \\
\frac{\left[x_{2}-x_{1} x_{1}^{\prime \prime}\right]}{\left[x_{1}^{\prime} x_{1}^{\prime \prime}\right]}=f_{3}(t), \frac{\left[x_{1}^{\prime} x_{2}-x_{1}\right]}{\left[x_{1}^{\prime} x_{1}^{\prime \prime}\right]}=f_{4}(t) .
\end{gathered}
$$

Proof. Assume that $x_{1}^{\prime}=y_{1}$ and $x_{2}-x_{1}=y_{2}$, then we have

$$
\begin{aligned}
& \frac{\left[y_{1} y_{1}^{\prime \prime}\right]}{\left[y_{1} y_{1}^{\prime}\right]}=f_{1}(t), \frac{\left[y_{1}^{\prime \prime} y_{1}^{\prime}\right]}{\left[\begin{array}{ll}
y_{1} & y_{1}^{\prime}
\end{array}\right]}=f_{2}(t), \\
& \left.\frac{\left[y_{2} y_{1}^{\prime}\right.}{\left[y_{1} y_{1}^{\prime}\right.}\right]=f_{3}(t), \frac{\left[y_{1} y_{2}\right.}{\left[y_{1} y_{1}^{\prime}\right]}=f_{4}(t) .
\end{aligned}
$$

On the other hand, assume that 


$$
A_{y_{1}}=\left(\begin{array}{ll}
y_{11} & y_{11}^{\prime} \\
y_{12} & y_{12}^{\prime}
\end{array}\right), \quad A_{y_{1}}^{\prime}=\left(\begin{array}{ll}
y_{11}^{\prime} & y_{11}^{\prime \prime} \\
y_{12}^{\prime} & y_{12}^{\prime \prime}
\end{array}\right)
$$

and $A_{y_{1}}^{-1} A_{y_{1}}^{\prime}=B$. Since $A_{y_{1}}^{\prime}=A_{y_{1}} B$, we obtain following equation system:

$$
\begin{aligned}
& y_{11}^{\prime}=b_{11} y_{11}+b_{21} y_{11}^{\prime} \\
& y_{12}^{\prime}=b_{11} y_{12}+b_{21} y_{12}^{\prime} \\
& y_{11}^{\prime}=b_{12} y_{11}+b_{22} y_{11}^{\prime} \\
& y_{12}^{\prime}=b_{12} y_{12}+b_{22} y_{12}^{\prime} .
\end{aligned}
$$

From this equation system, the following solutions are obtained:

$$
b_{11}=0, \quad b_{21}=1, \quad b_{12}=\frac{\left[y_{1}^{\prime \prime} y_{1}^{\prime}\right]}{\left[y_{1} y_{1}^{\prime}\right]}, \quad b_{22}=\frac{\left[y_{1} y_{1}^{\prime \prime}\right]}{\left[\begin{array}{ll}
y_{1} & y_{1}^{\prime}
\end{array}\right]} .
$$

In that case, we have the matrix $B$ as follows:

$$
\left(\begin{array}{ll}
0 & f_{2}(t) \\
1 & f_{1}(t)
\end{array}\right)
$$

Since $A_{y_{1}}^{\prime}=A_{y_{1}} B$, we have

$$
\begin{aligned}
& y_{11}^{\prime \prime}=f_{2}(t) y_{11}+f_{1}(t) y_{11}^{\prime} \\
& y_{12}^{\prime \prime}=f_{2}(t) y_{12}+f_{1}(t) y_{12}^{\prime} .
\end{aligned}
$$

Assume that $z=\left(\begin{array}{l}y_{11} \\ y_{12}\end{array}\right)$ then $z^{\prime \prime}=f_{1}(t) z^{\prime}+f_{2}(t) z$ is obtained. It's well-known that the last differential equation has at least one solution. Let the solution has the form $y_{1}(t)=\left(w_{1}(t), w_{2}(t)\right)$. Therefore the curve $y_{1}(t)$ satisfies the equations in (3). Consider the matrix $A_{2}=\left(\begin{array}{ll}y_{11} & y_{21} \\ y_{12} & y_{22}\end{array}\right)$ and assume that $A_{y_{1}}^{-1} A_{2}=K$. Hence we have $A_{2}=A_{y_{1}} K$ which leads to the following equation system:

$$
\begin{aligned}
& y_{11}=k_{11} y_{11}+k_{21} y_{11}^{\prime} \\
& y_{12}=k_{11} y_{12}+k_{21} y_{12}^{\prime} \\
& y_{21}=k_{12} y_{11}+k_{22} y_{11}^{\prime} \\
& y_{22}=k_{12} y_{12}+k_{22} y_{12}^{\prime} .
\end{aligned}
$$

From this equation system, the following solutions are obtained:

$$
k_{11}=1, \quad b_{21}=0, \quad k_{12}=\frac{\left[y_{2} y_{1}^{\prime}\right]}{\left[y_{1} y_{1}^{\prime}\right]}, \quad k_{22}=\frac{\left[y_{1} y_{2}\right]}{\left[y_{1} y_{1}^{\prime}\right]} .
$$

Namely, $K=\left(\begin{array}{cc}1 & f_{3}(t) \\ 0 & f_{4}(t)\end{array}\right)$. This let us write the following equation system:

$$
\begin{aligned}
& y_{21}=f_{3}(t) y_{11}+f_{4}(t) y_{11}^{\prime} \\
& y_{22}=f_{3}(t) y_{12}+f_{4}(t) y_{12}^{\prime} .
\end{aligned}
$$


By solving this equation system, a curve $y_{2}(t)=\left(u_{1}(t), u_{2}(t)\right)$ is obtained where $\operatorname{det}\left(\begin{array}{ll}u_{1}(t) & u_{2}(t) \\ u_{1}^{\prime}(t) & u_{2}^{\prime}(t)\end{array}\right) \neq 0$ and the curve $y_{2}(t)$ satisfies the equation (4). Since $x_{1}^{\prime}=y_{1}$ and $x_{2}-x_{1}=y_{2}$, two curves $x_{1}(t)$ and $x_{2}(t)$ are obtained and have the following form:

$$
\begin{aligned}
& x_{1}(t)=\int y_{1}(t) d t+c_{1} \\
& x_{2}(t)=y_{2}(t)+\int y_{1}(t) d t+c_{1} .
\end{aligned}
$$

This completes the proof.

\section{Conclusion and Future Work}

- This study could be generalized to the case of $n$-curves.

- On the other hand, studies in this paper could be transferred to 3- dimensional affine space.

- In that case, relation between surfaces and this study could be stated.

\section{REFERENCES}

[1] Liu, H., Curves in Affine and Semi-Euclidean Spaces, Results. Math., 65, 235-249, 2014.

[2] Sağıroğlu, Y., Affine Differential Invariants of Curves, Lambert Academic Publishing, Saarbrücken, 2012.

[3] Sağıroğlu, Y., Pekşen, Ö., The equivalence of centro-equiaffine curves, Turk. J. Math. 34, 95-104, 2010

[4] Sağıroğlu, Y., The equivalence problem for parametric curves in one-dimensional affine space, Int. Math. Forum, 6, 4, 177-184, 2011.

[5] Sağıroğlu, Y., The equivalence of curves in $\mathrm{SL}(\mathrm{n}, \mathrm{R})$ and its application to ruled surfaces, Appl. Math. Comput., 218, 1019-1024, 2011.

[6] Aripov, R.G., Khadjiev, D., The Complete System of Global Differential and Integral Invariants of a Curve in Euclidean Geometry, 51, 1-14, 2007.

[7] Sağıroğlu, Y., Yapar, Z., $G L(n, \mathbb{R})$-Equivalence of a Pair of Curves in Terms of Invariants, Journal of Mathematics and System Science, 6, 16-22, 2016.

[8] Su, B., Affine Differential Geometry, Science Press, Beijing, Gordon and Breache, New York, 1983.

[9] Schirokow P.A., Schirokow, A.P., Affine Differentialgeometrie, Teubner, Leipzig, 1962.

[10] Gardner, R.B., Wilkens, G.R., The Fundamental Theorems of Curves and Hypersurfaces in Centro-Affine Geometry, Bull. Belg. Math. Soc., 4, 379-401, 1997.

[11] Izumiya, S., Sano, T., Generic Affine Differential Geometry of Space Curves, Proceedings of the Royal Society of Edinburgh, 128A, 301-314, 1998. 